\title{
PET/CT Findings within the Contralateral Tonsil Following Unilateral Tonsillectomy
}

Nir Hirshoren $\mathrm{MD}^{1,2}$, Elizabeth Olayos $\mathrm{MD}^{1}$, Jason Callahan BAppSci ${ }^{3}$, Eddie Lau BPharm, $\operatorname{MBBS}^{3,4,5}$

${ }^{1}$ Division of surgical oncology, Peter MacCallum Cancer Center, Melbourne, Australia

${ }^{2}$ Department of Otolaryngology / Head \& Neck Surgery, Hebrew University School of Medicine

- Hadassah Medical Center, Jerusalem, Israel

${ }^{3}$ Division of radiation oncology and cancer imaging, Peter MacCallum Cancer Center,

Melbourne, Australia

${ }^{4}$ Department of Radiology, Austin Health, Melbourne, Australia

${ }^{5}$ Department of Radiology, Melbourne University, Australia

\section{A short running title: $\underline{\text { PET/CT Following Unilateral Tonsillectomy }}$}

Corresponding author: Nir Hirshoren, MD

Division of surgical oncology, Peter MacCallum Cancer Centre, Melbourne, Australia Postal address: Department of Otolaryngology/Head \& Neck Surgery, Hadassah Ein-

Kerem, Jerusalem, 91120, Israel

Tell: 972-2-6776469, Fax: 972-2-6435090

E-mail: drnir@hadassah.org.il

There are no financial or other interests to disclose.

This is the author manuscript accepted for publication and has undergone full peer review but has not been through the copyediting, typesetting, pagination and proofreading process, which may lead to differences between this version and the Version record. Please cite this article as doi:10.1002/ lary.26023. 


\section{Abstract:}

\section{Objectives:}

In the setting of considerable variation of pharyngeal palatine tonsil fluorine 18

fluorodeoxyglucose (FDG) uptake, we mostly rely on the asymmetry between the left and right sided tonsils to identify underlying malignancy.

The current study evaluates the FDG PET/CT findings within the contralateral tonsil among patients who had unilateral tonsillectomy and analyse the cancer incidence in the contralateral tonsil with high FDG avidity.

\section{Study Design:}

A retrospective cohort study in a single high volume tertiary cancer center.

\section{Methods:}

Analysis of the PET/CT scans performed at Peter MacCallum Cancer Center between $1^{\text {st }}$ June 2005 and $31^{\text {st }}$ December 2014.

\section{Results:}

The study group consists of 160 patients who underwent 308 post unilateral tonsillectomy FDG PET/CT scans. The majority of unilateral tonsillectomies were undertaken for the treatment or diagnosis of oropharyngeal squamous cell carcinoma $(85.1 \%)$.The mean follow-up time succeeding unilateral tonsillectomy was 38.2 months.

Ten $(6.25 \%)$ patients had relatively high contralateral tonsil FDG uptake (mean SUV MAX $_{6.9) .}$ Only one patient, with localized high FDG uptake but no other high uptake within the 
Waldeyer's ring and no previous PET/CT for comparison, underwent contralateral tonsillectomy.

This was found to be benign. None of the 160 patients, including those nine patients, had developed contralateral tonsil malignancy at the end of the study period.

\section{Conclusion:}

PET/CT remains a reliable surveillance tool within the contralateral tonsil following unilateral tonsillectomy. Comparison with previous PET/CT scans and comparison with other subsites within the Waldeyer's ring, in combination with appropriate follow-up and clinical judgment, facilitate PËT/CT high accuracy.
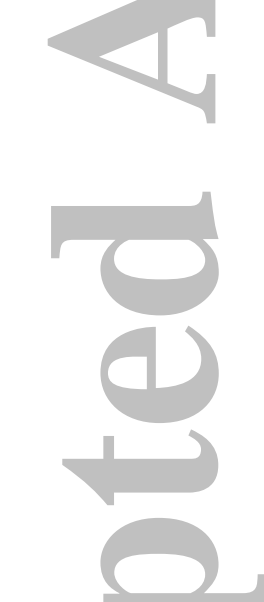

Key words: Tonsillectomy, PET/CT, Waldeyer's ring, Tonsil SCC, SCC of Unknown primary

Evidence based medicine: level 4

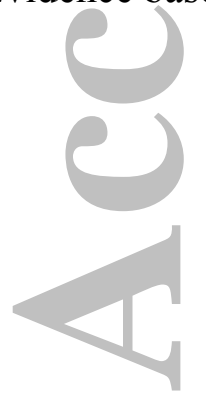




\section{Introduction:}

Unilateral tonsillectomy may potentially impair the accuracy of postoperative surveillance PET/CT scans within other lymphatic-rich Waldeyer's ring subsites, specifically within the contralateral tonsil. There is significant variation of pharyngeal palatine tonsil fluorine 18 fluorodeoxyglucose (FDG) uptake $(1,2)$, and thus we largely rely on the asymmetry between the left and right sided tonsils to identify underlying malignancy (3). Monitoring for asymmetry as a diagnostic strategy (4) is compromised following unilateral tonsillectomy. This relatively unexplored and potentially worrisome phenomenon is worthy of further consideration given the wide use of PET/CT among patients who undergo unilateral tonsillectomy for oropharyngeal squamous cell carcinoma (SCC) or as part of investigation of SCC of an unknown primary (SCCUP) of the head and neck region.

Certainly, the debate as to whether unilateral tonsillectomy is sufficient for the evaluation of unknown head and neck SCC, is unresolved, especially in view of the risk for synchronous tonsilar tumor $(5,6,7)$. Therefore, the potential negative impact of inaccurate surveillance PET scanning may be an additional reason to encourage many surgeons to opt for bilateral tonsillectomy over unilateral tonsillectomy, even in cases with obvious unilateral disease.

In the present study we investigate our null hypotheses that high FDG uptake in the contralateral tonsil following unilateral tonsillectomy is not a common clinical problem. Moreover, PET/CT scan remains a reliable tool for surveillance among these patients because of a low rate of unnecessary contralateral tonsillectomy. No contralateral tonsil cancer has developed in three years follow up. 


\section{Materials and Methods:}

A single tertiary center retrospective cohort study of the FDG PET/CT scan findings for surveillance within the Waldeyer's ring following unilateral tonsillectomy was conducted. The study was performed with the approval of the local ethical committee $(15 / 65 \mathrm{R})$ according to the World Medical Association Declaration of Helsinki 2008.

Inclusion criteria: all patients who had unilateral tonsillectomy (for oropharyngeal cancer or as part of SCCUP of the head and neck region investigation) followed by at least one PET/CT scan at Peter MacCallum Cancer Center between the $1^{\text {st }}$ of June 2005 and $31^{\text {st }}$ of December 2014 were included.

Exclusion criteria: cases in which the pathology report was not available for assessment (in order to exclude bilateral tonsillectomy or small biopsies called tonsillectomy) and cases in which PET/CT scans could not be reviewed or analysed were excluded.

Primary objectives: To evaluate the PET/CT findings within the contralateral tonsil among patients who had unilateral tonsillectomy and to analyse cancer incidence in contralateral highly avid tonsils.

PET/CT technique: All patients fasted for at least 6 hours prior to intravenous administration of 3.7 $\mathrm{MBq} \mathrm{kg}^{-1}$ body weight of ${ }^{18}$ F-FDG. The ${ }^{18}$ F-FDG study was performed with on dedicated PET/CT systems (Discovery ST and V690, GE Medical Systems, Waukesha, WI; Biograph 64, Siemens). One hour after ${ }^{18}$ F-FDG injection, each patient underwent a single, integrated PET/CT examination. Patients were instructed to breath freely for the duration of the test. A low-dose CT scan was acquired from the vertex to the proximal thighs. All PET/CT images were clinically reviewed on a Siemens True-D workstation by experienced PET nuclear medicine physicians or 
radiologists and study reports were available on medical record. The PET scan reports of all PET/CT studies were retrospectively evaluated by two investigators. The PET/CT images of the cases with high contralateral tonsil uptake reported were retrieved and analysed qualitatively and quantitatively on the MIMvista software (MIM-5.4, MIM Software Inc, Cleveland, OH) by an expert radiologist (EL). The lesion of interest was contoured using the PET Edge contouring tool and the maximal and mean standardized uptake values (SUVmax and SUVmean) were recorded.

Statistics: Basic patient demographics, disease characteristics, radiation treatment, as well as the SUVmax and SUVmean within the regions of interest are summarized using means, medians and ranges when appropriate.

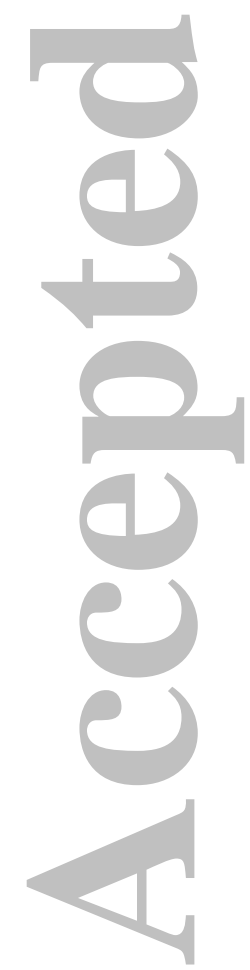




\section{$\underline{\text { Results: }}$}

Overall, 160 patients had unilateral tonsillectomy followed by at least one PET/CT scan during the study period. The majority $(84.4 \%)$ were men, the average age was 58.6 years old and the tonsillectomy side was nearly equally distributed. Most unilateral tonsillectomies done for the treatment or diagnosis of oropharyngeal SCC (85.1\%), followed by benign specimens $(11.2 \%)$ as part of the SCCUP investigation, lymphoma (3.1\%) and melanoma (0.6\%). The radiation details of 153 patients $(95.6 \%)$ were available for analysis. Three patients had no radiation treatment following the tonsillectomy. The median radiation dose was 70 Gray provided to one side or both sides of the neck in $51.35 \%$ and $48.65 \%$ of the patients respectively. The mean follow-up time following unilateral tonsillectomy was 38.2 months (table 1).

Most patients (mean) had two PET/CT scans following unilateral tonsillectomy, ranging between 1 to 9 scans. In total, 308 scans were performed on average 6.85 months following the unilateral tonsillectomy. Ten (6.25\%) patients had qualitative description of high contralateral tonsil FDG uptake. The average contralateral tonsil $\mathrm{SUV}_{\mathrm{MAX}}$ in this subgroup was 6.9, ranged between 4.3 and 10.5. Of those 10 patients, seven also had relatively high $\mathrm{SUV}_{\mathrm{MAX}}$ tongue base avidity. Those seven patients had a Waldeyer's ring diffuse FDG uptake pattern on qualitative assessment. Overall, the mean $\mathrm{SUV}_{\mathrm{MAX}}$ within the tongue base was 4.5 , ranging between 2.6 and 7.53. Four of the 10 patient (40\%) with contralateral high FDG uptake had pre-operative PET/CT scans demonstrating the same qualitative high FDG uptake within the contralateral tonsil. (table 2)

One patient, with localized, high FDG uptake (figure 1a) within the contralateral tonsil (SUV MAX 10.5) without any other uptake elsewhere in the Waldeyer's ring (tongue base SUV $\mathrm{MAX}_{2.6)}$ and 
no previous PET/CT scan done for comparison, had contralateral tonsillectomy. The contralateral tonsil specimen did not demonstrate cancer on histology. The other nine patients had clinical follow-up and/or repeated PET/CT scans and none of these patients developed tonsil cancer during 27 months (mean) follow up period.

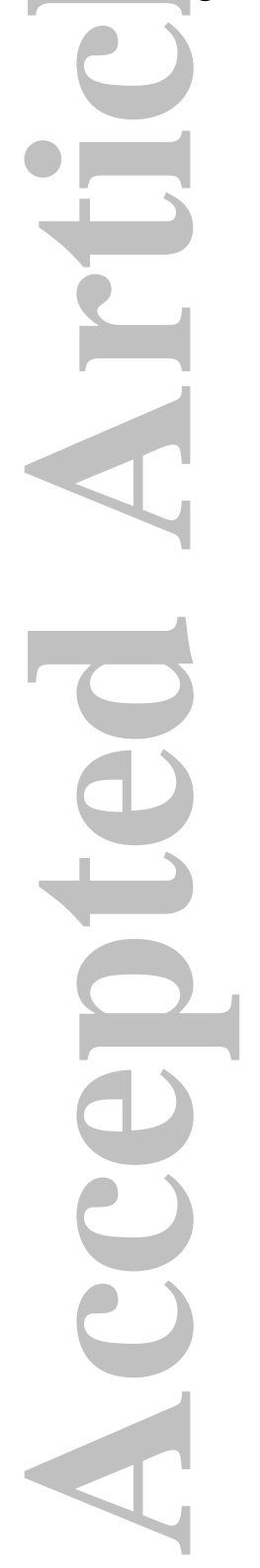




\section{Discussion:}

Tonsillectomy gives rise to potential anatomical (3) and metabolic alterations, which could be problematic in ongoing surveillance. In this paper, we describe the FDG uptake within the contralateral tonsil and Waldeyer's ring, particularly the tongue base, following unilateral tonsillectomy, investigating PET/CT accuracy and efficacy in the post unilateral tonsillectomy setting. This is becoming increasingly important, considering the rising incidence of SCC of the tonsil and other oropharyngeal subsites, which may be related to the recognized association with HPV infection over and above "traditional" risk factors such as alcohol abuse and smoking. (8) Intense physiological FDG uptake within the palatine tonsil is a well-known phenomenon, established from studies investigating oral cavity (9) and other head and neck malignancies (10), in addition to non head and neck cancers (11). Symmetrical FDG uptake within the Waldeyer's ring is one of the main methods distinguishing benign and suspicious malignant tumors (3), therefore, unilateral tonsillectomy may potentially diminish the accuracy of PET/CT in ongoing surveillance of these patients.

In our study population, only a small portion of PET/CT scans conducted following unilateral tonsillectomy $(6.25 \%$ of the patients) demonstrated relatively high FDG avid within the contralateral tonsil. In most cases (9 out of 10 patients), contralateral tonsillectomy was avoided by employing clinical judgment, comparison to different subsites of the Waldeyer's ring (particularly the tongue base) and reviewing previous scans. Most patients in this study with relatively high FDG uptake within the contralateral tonsil (70\%) had diffuse (qualitative assessment) and relatively high uptake (quantitative measurement) within other subsites of Waldeyer's ring (figure 1b). Moreover, 40\% had the same contralateral FDG uptake before having the unilateral tonsillectomy of the more intensely avid tonsil. Only one of the 160 patients 
$(0.6 \%)$ had contralateral tonsillectomy revealing a benign hypertrophic tonsil. All other patients, including the 9 patients with high contralateral uptake, had no evidence of Waldeyer's ring (including contralateral tonsil) cancer development during a mean of greater than 3 years follow up.

Tonsillectomy is a crucial step in the treatment (12) and investigation of oropharyngeal cancers and SCCUP (13). The bleeding risk (around 5\%) following tonsillectomy among adults (14) may support the practice of avoiding bilateral tonsillectomy when not required. Pain control, dehydration and fever, although less hazardous, are expected to be of less significance following unilateral (compared to bilateral) tonsillectomy. (14)

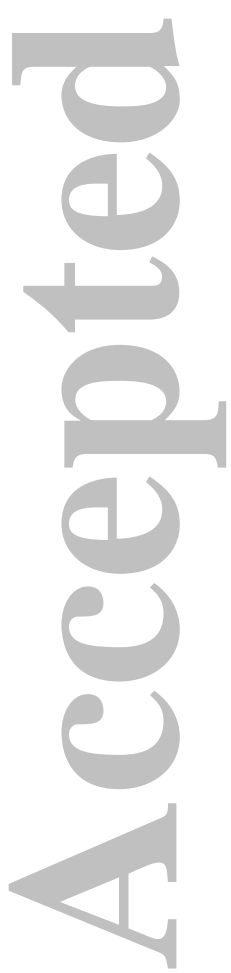




\section{Conclusion:}

According to our study results, PET/CT scan surveillance within Waldeyer's ring remains accurate following unilateral tonsillectomy and therefore presumed difficulty in the interpretation of post-tonsillectomy FDG PET scan is not a valid reason for performing bilateral tonsillectomy routinely.
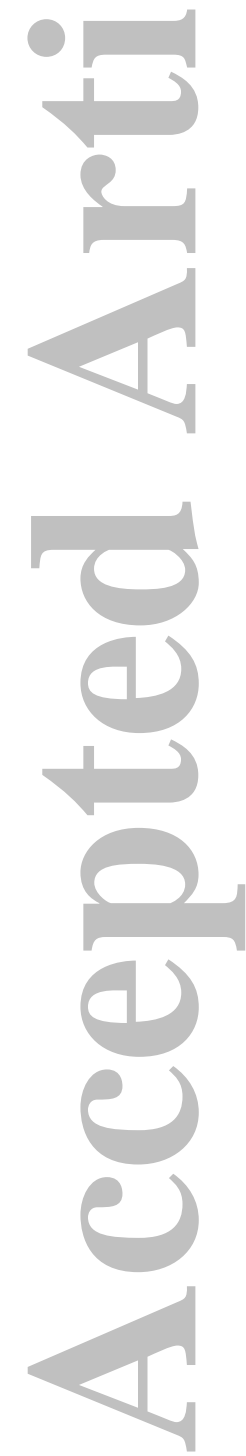


\section{$\underline{\text { References: }}$}

1. Kawabe J, Okamura T, Shakudo M et al. Physiological FDG uptake in the palatine tonsils. Ann Nucl Med 2001;15(3):297-300

2. Heusner TA, Hahn S, Hamami ME et al. Incidental head and neck (18)F-FDG on PET/CT without corresponding morphological lesion: early predictor of cancer development? Eur J Nucl Med Mol imaging 2009;36(9):1397-406

3. Wong WL, Gibson D, Sanghera B et al. Evaluation of normal FDG uptake in palatine tonsil and its potential value for detecting occult head and neck cancers: a PET CT study. Nucl Med Commun 2007; 28(9):675-80

4. Marcio R. T. Garcia, Ula L. Passos, Tamara A. Ezzedine, Henrique B. Zuppani, Regina L. E. Gomes, Eloisa M. S. Gebrim. Postsurgical Imaging of the Oral Cavity and Oropharynx: What Radiologists Need to Know. Radiographics 2015;35(3):804-18

5. Kothari P, Randhawa PS, Farrell R. Role of tonsillectomy in the search for a squamous cell carcinoma from an unknown primary in the head and neck. Br J Oral Maxillofac Surg 2008 $46(4): 283-7$

6. Roeser MM, Alon EE, Olsen KD. Synchronous bilateral tonsil squamous cell carcinoma. Laryngoscope 2010; 120 suppl 4:S181

7. Wayne M. Koch, Nassir Bhatti, Mark F. Williams, David W. Eisele, Oncologic Rationale for Bilateral Tonsillectomy in Head and Neck Squamous Cell Carcinoma of Unknown Primary Source. Otolaryngology Head Neck Surg 2001;124(3):331-3

8. Maxwell JH, Grandis JR, Ferris RL. HPV-Associated Head and Neck Cancer: Unique Features of Epidemiology and Clinical Management. Annu Rev Med. 2016;14(67):91-101. 
9. S Kito, H Koga, M Kodama, et al. Variety and complexity of fluorine-18-labelled fluoro-2deoxy-D-glucose accumulations in the oral cavity of patients with oral cancers. Dentomaxillofac Radiol. 2013; 42(7):1-8

10. Nakamura S, Okochi K, Murata Y, Shibuya H, Kurabayashi T. [18F]FluorodeoxyglucosePET/CT differentiation between physiological and pathological accumulations in head and neck. Nucl Med Commun. 2009;30(7):498-503.

11. Nakamoto Y, Tatsumi M, Hammoud D, Cohade C, Osman MM, Wahl RL. Normal FDG distribution patterns in the head and neck: PET/CT evaluation. Radiology. 2005;234(3):879-85.

12. Rahmati R, Dogan S, Pyke O et al. Squamous cell carcinoma of the tonsil managed by conventional surgery and postoperative radiation. Head Neck 2015;37(6):800-807

13. Straetmans J, Vent J, Lacko M et al. Management of neck metastases of unknown primary origin united in two European centers. Eur Arch of Otorhinolaryngol 2015;272(1):195-205 14. Bhattacharyya N. Healthcare disparities in revisits for complications after adult tonsillectomy. Am j of otolaryngol 2015;36(2):249-253 


\section{Figure Legends:}

Figure 1:

Different patterns of FDG uptake following unilateral tonsillectomy.

Figure 1a - Upper panel: right image axial CT, left image axial PET; lower panel: right image axial fused PET/CT, left image Maximum Intensity Projection MIP. PET High FDG uptake within the left contralateral tonsil (white arrow) following unilatreral right tonsillectomy. Figure $1 \mathrm{~b}$-Diffuse Waldeyer's ring uptake including the right contralateral tonsil (white arrow) and tongue base (white dashed arrow) following unilateral left tonsillectomy

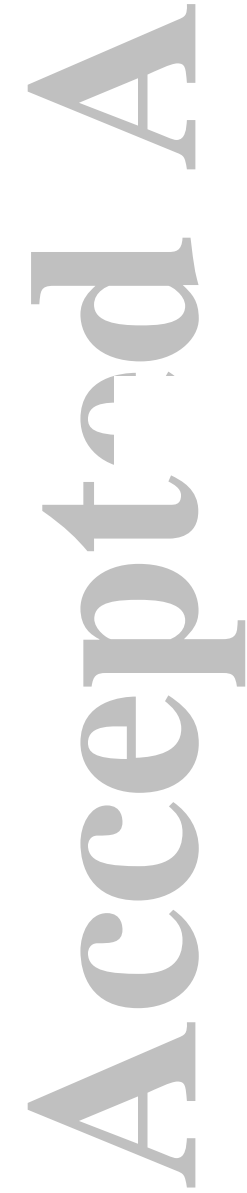


Table 1: Demographic and treatment description of study population

\begin{tabular}{|c|c|c|}
\hline Age & & $\mathrm{N}=160$ \\
\hline & Average (range), years & $58.6(34.1-85.5)$ \\
\hline & Median, years & 59.3 \\
\hline \multicolumn{3}{|c|}{ Gender } \\
\hline & Female (percentage) & $25(15.6 \%)$ \\
\hline & Male (percentage) & $135(84.4 \%)$ \\
\hline \multicolumn{3}{|c|}{ Side of tonsillectomy } \\
\hline & Right & $79(49.4 \%)$ \\
\hline & Left & $81(50.6 \%)$ \\
\hline \multicolumn{3}{|c|}{ Histology analysis of tonsil tissue } \\
\hline & Squamous Cell Carcinoma & $136(85.1 \%)$ \\
\hline & No tumor & $18(11.2 \%)$ \\
\hline & Lymphoma & $5(3.1 \%)$ \\
\hline & Melanoma & $1(0.6 \%)$ \\
\hline \multicolumn{2}{|c|}{ Radiation treatment } & $\mathrm{N}=153$ \\
\hline \multirow[t]{2}{*}{ Dose } & Average (range), gray & $66.4(20-73)$ \\
\hline & Median & 70 \\
\hline
\end{tabular}




\begin{tabular}{lll}
\hline Fields & $\mathrm{N}=148$ \\
\hline & Unilateral & $76(51.35 \%)$ \\
\hline & & $72(48.65 \%)$ \\
\hline
\end{tabular}

Follow up period following

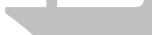

unilateral tonsillectomy

\begin{tabular}{lll} 
& \\
\hline & Average (range), months & $38.2(0.7-121.6)$ \\
\hline
\end{tabular}

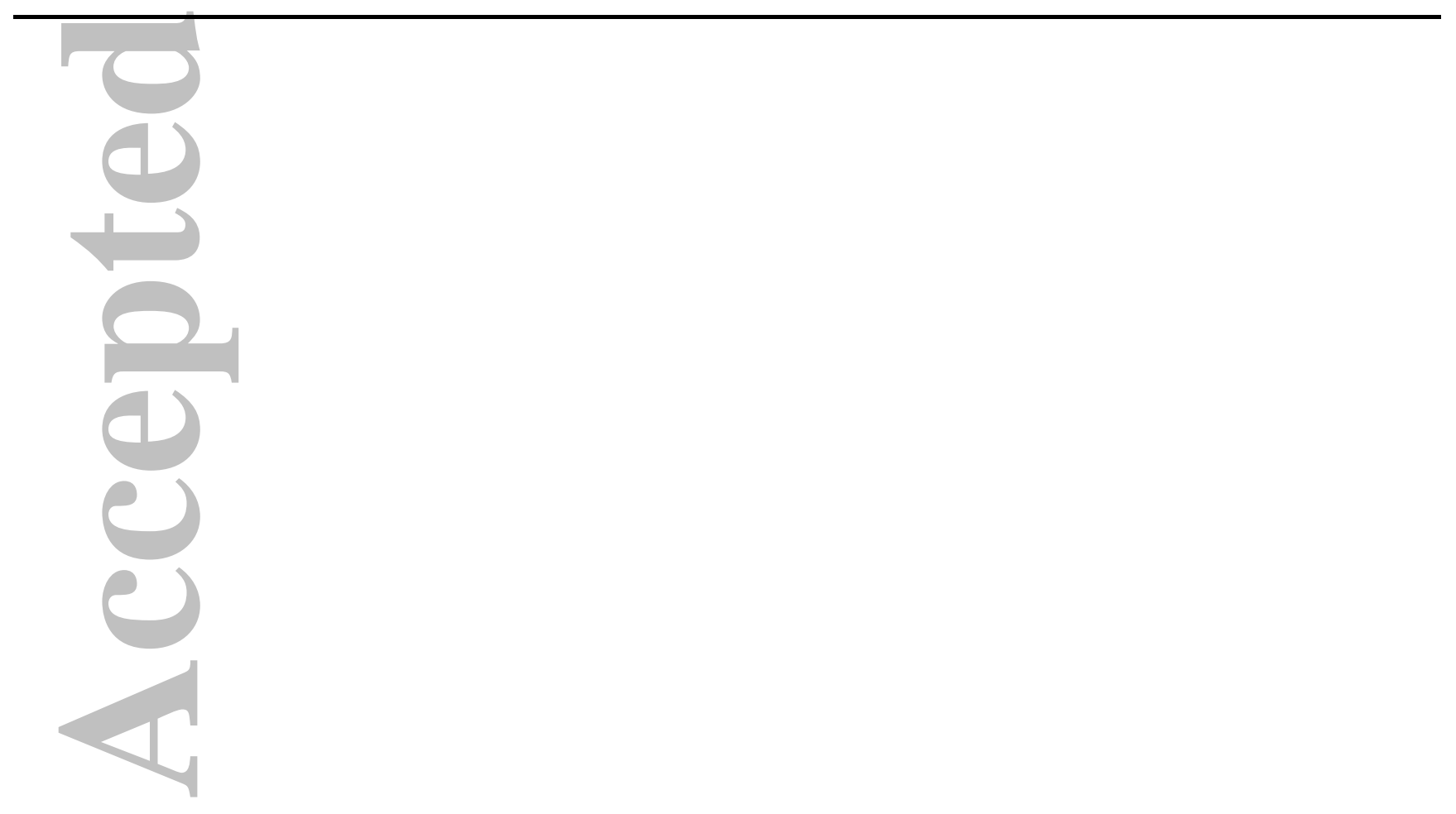

John Wiley \& Sons

This article is protected by copyright. All rights reserved. 
Table 2: PET FDG scan description

\begin{tabular}{|c|c|c|}
\hline \multicolumn{2}{|c|}{ PET scans done following unilateral tonsillectomy } & \multirow{2}{*}{$\begin{array}{l}N=308 \\
2(1-9)\end{array}$} \\
\hline & Median (range) scans per patient & \\
\hline & $\begin{array}{l}\text { Average time between first PET scan and unilateral tonsillectomy } \\
\text { (range), months }\end{array}$ & $6.85(0.03-109.9)$ \\
\hline & Median time between first PET scan and tonsillectomy, months & 5.3 \\
\hline \multicolumn{2}{|c|}{ PET scans demonstrating high FDG uptake within contralateral tonsil } & $\mathrm{N}=10(6.25 \%)$ \\
\hline \multirow{2}{*}{$\begin{array}{l}\text { Average (range) } \\
\text { highest SUV count }\end{array}$} & within contralateral tonsil & $6.9(4.3-10.5)$ \\
\hline & within the Waldeyer's ring & $4.5(2.6-7.53)$ \\
\hline \multirow{2}{*}{ Median highest SUV } & within contralateral tonsil & 6 \\
\hline & within the Waldeyer's ring & 4 \\
\hline \multirow{2}{*}{$\begin{array}{l}\text { Average (range) } \\
\text { mean SUV count }\end{array}$} & within contralateral tonsil & $4.9(3-7.3)$ \\
\hline & within the Waldeyer's ring & $3.5(2.1-6.1)$ \\
\hline \multirow{2}{*}{ Median mean SUV } & within contralateral tonsil & 4.5 \\
\hline & within the Waldeyer's ring & 3.2 \\
\hline \multirow[t]{2}{*}{+1} & $\begin{array}{l}\text { Number of patients (percentage) with high FDG uptake (quantitative) } \\
\text { within the Waldeyer's ring apart from the contralateral tonsil }\end{array}$ & $7(70 \%)$ \\
\hline & Number of patients (percentage) with diffuse (qualitative) high FDG & $7(70 \%)$ \\
\hline
\end{tabular}


uptake within the Waldeyer's ring apart from the contralateral tonsil

Number of patients (percentage) with high FDG uptake within the 4 (40\%)

contralateral tonsil prior unilateral tonsillectomy

Number of patients having contralateral tonsillectomy, (percentage) $1(0.6 \%)$

\begin{tabular}{|c|c|c|}
\hline \multirow{2}{*}{$\begin{array}{l}\text { Follow up period, } \\
\mathrm{N}=10\end{array}$} & Average (range), months & $27(9.8-57.2)$ \\
\hline & Median, months & 26.2 \\
\hline & Incidence of contralateral tonsil SCC & 0 \\
\hline
\end{tabular}

SUV: Standardized Uptake Value 


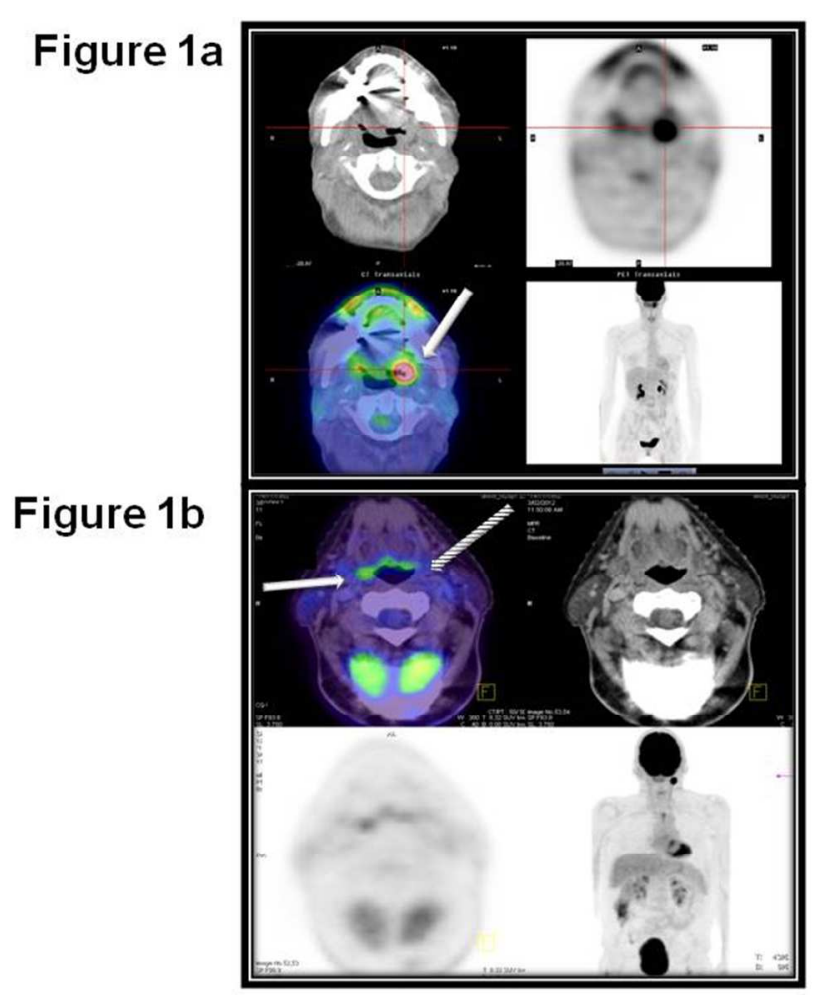

Figure 1: Different patterns of FDG uptake following unilateral tonsillectomy. Figure 1a - Upper panel: right image axial CT, left image axial PET; lower panel: right image axial fused PET/CT, left image Maximum Intensity Projection MIP. PET High FDG uptake within the left contralateral tonsil (white arrow) following unilatreral right tonsillectomy. Figure $1 \mathrm{~b}$ - Diffuse Waldeyer's ring uptake including the right contralateral tonsil (white arrow) and tongue base (white dashed arrow) following unilateral left tonsillectomy

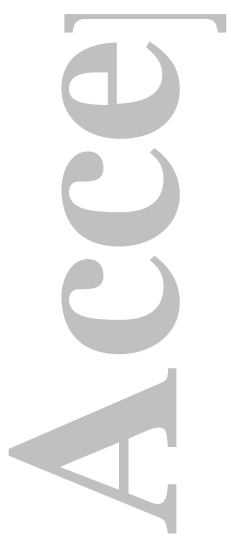
$254 \times 190 \mathrm{~mm}$ (96 x 96 DPI) 


\section{University Library}

\section{- M M N E R VA A gateway to Melbourne's research publications}

Minerva Access is the Institutional Repository of The University of Melbourne

Author/s:

Hirshoren, N;Olayos, E;Callahan, J;Lau, E

Title:

PET/CT findings within the contralateral tonsil following unilateral tonsillectomy

Date:

2016-11-01

Citation:

Hirshoren, N., Olayos, E., Callahan, J. \& Lau, E. (2016). PET/CT findings within the contralateral tonsil following unilateral tonsillectomy. LARYNGOSCOPE, 126 (11), pp.2480-2483. https://doi.org/10.1002/lary.26023.

Persistent Link:

http://hdl.handle.net/11343/291213 